Gerinnungsmonitor hat sich bewährt

\title{
Schnelle und verlässliche INR-Messung für Ärzte und Patienten
}

— Die Firma Alere feiert ein Jubiläum: der kleine Gerinnungsmonitor INRatio ${ }^{\circledR}$ liefert seit zehn Jahren verlässliche INR-Ergebnisse für antikoagulierte Patienten. Das Point-of-Care-System ist beliebt und wird häufig genutzt.

Die neueste Generation, der Alere INRatio ${ }^{\oplus} 2$ Gerinnungsmonitor, überzeugt Ärzte und Patienten vor allem durch seine Messgenauigkeit, seine einfache Bedienung und die mitgeführten Kontrollen, die ein umständliches Hantieren mit Kontrollflüssigkeiten unnötig macht. Mit jedem Test werden automatisch zwei quantitative Qualitätskontrollen auf den Teststreifen durchgeführt. Die Bestimmung der TPZ/ INR erfolgt in nur 60 Sekunden.

Der Gerinnungsmonitor eignet sich aufgrund seiner leichten Bedienbarkeit auch für das Patienten-Selbstmanagement. Die Daten lassen sich auf den Computer übertragen, und das System speichert 120 Testergebnisse mit Datum und Zeit. Mit dem Übertragungsmodul CLAconnect ist eine
Integration in telemedizinische Betreuungszentren möglich.

Die einzeln verpackten und bei Raumtemperatur lagerbaren INRatio ${ }^{\circledR 2}$ Teststreifen enthalten eine Heparin-neutralisierende Substanz und ermöglichen eine zuverlässige Bestimmung der TPZ/INR auch während der Bridgingtherapie mit Heparin. Die Teststreifen sind unempfindlich gegenüber Heparin bis zu einer Konzentration von $3 \mathrm{U} / \mathrm{ml}$ und damit gut geeignet für das Monitoring bei HeparinBridging. Fondaparinux (bis $5 \mathrm{mg} / \mathrm{l}$ ), Acetylsalicylsäure (bis $4 \mathrm{mmol} / \mathrm{l}$ ), Clopidogrel (bis $20 \mathrm{mg} / \mathrm{dl}$ ) und Atorvastatin (bis 600 $\mu \mathrm{g} / \mathrm{l}$ ) haben ebenfalls keinen Einfluss auf die Messergebnisse.

Ärzte können die Bestimmung der Thromboplastinzeit mittels Teststreifen nach EBM-Ziffer 32026 TPZ (Quickwert, INR) mit 4,70 Euro abrechnen.

- Red.

Quelle: Nach Informationen von Alere
Kurz notiert

Zulassungserweiterung für Antidiabetika - Für Typ-2-Diabetiker, die trotz gesteigerter Bewegung, Ernährungsumstellung und einer eingeleiteten medikamentösen Behandlung ihre angestrebten Blutzuckerwerte nicht erreichen, gibt es neue Therapieoptionen. Galvus $^{\circledR}$ (Vildagliptin) und Eucreas ${ }^{\circledast}$ (Vildagliptin und Metformin) wurden in Kombination mit anderen Therapeutika zugelassen. Die Europäische Kommission erteilte erweiterte $\mathrm{Zu}$ lassungen

_ für Vildagliptin, mit oder ohne Metformin, in Kombination mit einer stabilen Dosis Insulin sowie

- für die Kombinationstherapie von Vildagliptin mit einem Sulfonylharnstoff und Metformin.

Die beiden Zulassungserweiterungen basieren auf den positiven CHMPBeurteilungen, die aufgrund überzeugender Ergebnisse aus zwei Parallelgruppenstudien ausgesprochen wurden.

Novartis

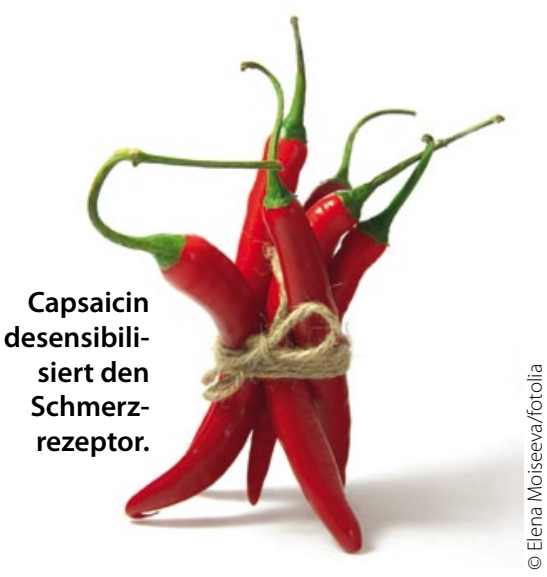

ergeben. Die enzymatische Aktivität der Cyclooxygenase sei um 70-75\% gehemmt worden. Die enthaltene Kermesbeere wirke ebenfalls antiphlogistisch über die in ihr enthaltenen Saponine und Lektine. Zusätzlich weise sie immunmodulierende Eigenschaften auf.

- Red.

Quelle: Nach Informationen von Bionorica SE 